\title{
Effects of peritumoral bevacizumab injection against oral squamous cell carcinoma in a nude mouse xenograft model: A preliminary study
}

\author{
HISATO YOSHIDA ${ }^{1}$, HITOSHI YOSHIMURA ${ }^{1}$, SHINPEI MATSUDA ${ }^{1}$, TAKASHI RYOKE ${ }^{1}$, \\ TAMOTSU KIYOSHIMA ${ }^{2}$, MOTOHIRO KOBAYASHI ${ }^{3}$ and KAZUO SANO ${ }^{1}$ \\ ${ }^{1}$ Department of Dentistry and Oral Surgery, Unit of Sensory and Locomotor Medicine, \\ Division of Medicine, Faculty of Medical Sciences, University of Fukui, Fukui 910-1193; ${ }^{2}$ Laboratory of \\ Oral Pathology, Division of Maxillofacial Diagnostic and Surgical Sciences, Faculty of Dental Science, \\ Kyushu University, Fukuoka 812-8582; ${ }^{3}$ Department of Tumor Pathology, Unit of Pathological Sciences, \\ Division of Medicine, Faculty of Medical Sciences, University of Fukui, Fukui 910-1193, Japan
}

Received September 17, 2017; Accepted January 19, 2018

DOI: $10.3892 / \mathrm{ol} .2018 .8399$

\begin{abstract}
Angiogenesis serves a crucial role in tumor growth. Vascular endothelial growth factor (VEGF) is a potent regulator of tumor angiogenesis and is highly expressed in oral squamous cell carcinoma (OSCC). Bevacizumab, which binds to VEGF-A, inhibits the biological activity of VEGF and is clinically administered by intravenous injection. As intravenous chemotherapy intensifies the side effects experienced by OSCC patients, an alternative treatment option is desirable, particularly for older patients with OSCC who present with systemic disease complications. Generally, local injections of antitumor agents enhance tumoricidal activity and decrease side effects. However, the antitumor effects of peritumoral bevacizumab injections in OSCC are not fully understood. Therefore, the present study examined the effects of peritumoral bevacizumab injections in an experimental nude mouse model of OSCC through immunohistochemical staining for cluster of differentiation (CD) 31 and $\alpha$-smooth muscle actin $(\alpha$-SMA) and apoptosis assays. It was identified that peritumoral injections of bevacizumab significantly inhibited tumor growth in OSCC xenografts compared with peritumoral saline injections or no treatment (controls), and it was also revealed that treatment with bevacizumab significantly reduced CD31- and $\alpha$-SMA-positive microvessel density $(\mathrm{P}<0.01)$ and increased level of tumor cell apoptosis $(\mathrm{P}<0.01)$ compared with the controls. In conclusion, these results collectively support
\end{abstract}

Correspondence to: Dr Kazuo Sano, Department of Dentistry and Oral Surgery, Unit of Sensory and Locomotor Medicine, Division of Medicine, Faculty of Medical Sciences, University of Fukui, 23-3 Matsuokashimoaizuki, Eiheiji, Fukui 910-1193, Japan

E-mail:sano@u-fukui.ac.jp

Key words: oral squamous cell carcinoma, angiogenesis, vascular endothelial growth factor, bevacizumab, peritumoral injection the experimental basis for the clinical development of peritumoral bevacizumab injections for the treatment of OSCC.

\section{Introduction}

Oral squamous cell carcinoma (OSCC) is the most common malignancy arising in the head and neck globally (1). Over the previous decades, the incidence of OSCC has significantly increased, and only a small improvement in the 5-year survival rate has been demonstrated (from 56\% in 1989-1994 to 62\% in 2007-2011) in a Netherlands study between 1989 and 2011, despite advances in surgical techniques, radiation and chemotherapy (2). It is widely accepted that angiogenesis serves an important role in tumor growth and is one of the hallmarks of malignant tumors, including OSCC (3-5). Therefore, inhibiting angiogenesis provides opportunities for the development of novel therapeutic agents and strategies for OSCC.

Vascular endothelial growth factor (VEGF) is one of the potent regulators of tumor angiogenesis and is considered to be an important therapeutic target $(4,5)$. VEGF-A is a key VEGF family member that is known to be involved in tumor angiogenesis in a number of malignancies $(6,7)$. Several groups have indicated that marked VEGF-A expression is observed in $70 \%$ of OSCC tissues (8), and that its expression levels are significantly increased in patients with OSCC with lymph node metastasis or a poor prognosis compared with those with a good prognosis (9-11).

One of the well-recognized VEGF-targeting drugs is bevacizumab, a recombinant humanized monoclonal antibody against VEGF-A, which inhibits the biological activity of VEGF (12). It is clinically administered by intravenous injection. Previous studies have indicated that the preclinical and clinical use of bevacizumab has beneficial effects on a variety of malignant tumors, including metastatic colorectal (13), breast (14) and lung cancer (15). In addition, the antitumor effects of bevacizumab have been demonstrated in nude mouse xenograft models of head and neck squamous cell carcinoma (HNSCC) (16-19). 
Generally, as life expectancy extends, the number of elderly patients ( $\geq 65$ years) with HNSCC increases (20). This results in difficulties in treatment, as intravenous chemotherapy against HNSCC, including OSCC, intensifies the side effects experienced by elderly patients (21). Antitumor agents may be delivered directly into tumor tissues by local injection. These local injections enhance the antitumor activity and diminish the side effects of the chemotherapeutic agents. Intratumoral injections, representing one of multiple local injection techniques, have already been established in the treatment of lung cancer (22). Intratumoral injections of bevacizumab were indicated to reduce tumor growth in squamous cell carcinoma (SCC) xenografts (19). However, the antitumor effects of peritumoral bevacizumab injection in mouse models of SCC, including OSCC, are not well understood. Therefore, the present study investigated the antitumor effects of peritumoral bevacizumab injections on OSCC xenografts. To the best of our knowledge, this is the first study to demonstrate the effects of peritumoral bevacizumab injections on OSCC.

\section{Materials and methods}

Reagents. Bevacizumab was purchased from Chugai Pharmaceutical Co., Ltd. (Tokyo, Japan) and dissolved in saline. Rat anti-mouse cluster of differentiation (CD)31 monoclonal antibody was purchased from DIANOVA Vertriebs Gesellschaft mbH (Hamburg, Germany). Anti- $\alpha$-smooth muscle actin ( $\alpha$-SMA) was purchased from Abcam (Cambridge, MA, USA). Indigocarmine was purchased from Daiichi Sankyo Company (Tokyo, Japan). All other chemicals and reagents were purchased from Invitrogen; Thermo Fisher Scientific, Inc. (Waltham, MA, USA), Sigma-Aldrich; Merck KGaA (Darmstadt, Germany) and Wako Pure Chemical Industries, Ltd. (Osaka, Japan), unless otherwise specified.

Cell lines and culture conditions. The HSC-3 cell line (Japanese Cancer Research Resources Bank, Tokyo, Japan), a human OSCC cell line, was used in the present study. The cells were maintained in $\alpha$-minimum essential medium ( $\alpha$-MEM) (Invitrogen; Thermo Fisher Scientific, Inc.) supplemented with $10 \%$ fetal bovine serum (BioWest, Nuaillé, France), $100 \mathrm{IU} / \mathrm{ml}$ penicillin and $100 \mathrm{mg} / \mathrm{ml}$ streptomycin (Invitrogen; Thermo Fisher Scientific, Inc.) and grown at $37^{\circ} \mathrm{C}$ in a $5 \% \mathrm{CO}_{2}$ atmosphere.

OSCC treatment model. A total of 18 female BALB/c nude $(n u / n u)$ mice weighing 18.9 $\pm 1.0 \mathrm{~g}$ (5-week-old; CLEA Japan, Inc., Tokyo, Japan) were used in the present study. All mice were kept in housing conditions of $21-25^{\circ} \mathrm{C}$ and $40-70 \%$ humidity, in a $12 \mathrm{~h}$ dark/light cycle with free access to food and water. A total of $5 \times 10^{6} \mathrm{HSC}-3$ cells were re-suspended in $50 \mu \mathrm{l} \alpha$-MEM mixed with $50 \mu \mathrm{l}$ of Matrigel (BD Biosciences, San Jose, CA, USA; non-diluted), and injected subcutaneously into the flank of anesthetized mice. Tumor-bearing mice were randomly divided into three groups ( $\mathrm{n}=6$ each), and tumors were allowed to reach $\sim 40 \mathrm{~mm}^{3}$ in size. Weight loss exceeding $20 \%$ was considered a humane endpoint.

The model in the present study used peritumoral injection, not intratumoral, to avoid artificial injury by the needle and to surround the tumor mass with the injected solution. The

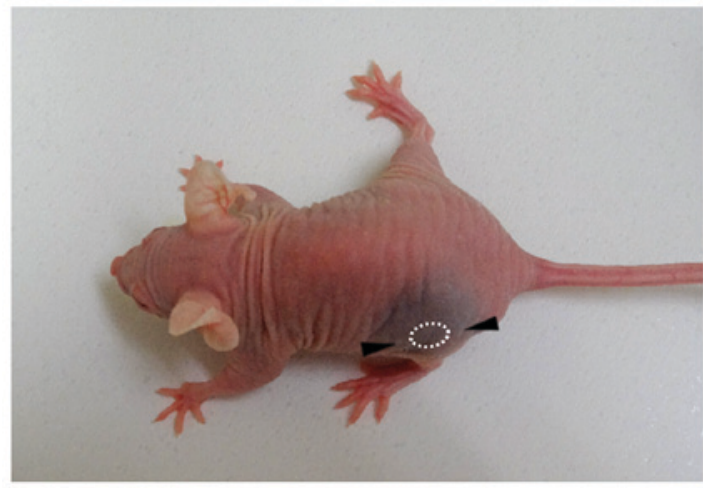

Figure 1. Image of a mouse following peritumoral injection of indigocarmine in a preliminary experiment. Indigocarmine was subcutaneously injected a distance of $2 \mathrm{~mm}$ from the tumor periphery into the caudal and rostral sides, respectively (as indicated by the arrowheads). The tissue around the tumor was stained blue.

following method was demonstrated to be most suitable for spreading the injected solution around the tumor. A Hamilton microliter syringe (50 $\mu \mathrm{l}$; Hamilton Company, Reno, NV, USA) with a 26-gauge needle was used. The needle was inserted subcutaneously a distance of $5 \mathrm{~mm}$ from the tumor periphery into the caudal-rostral side, positioned to a distance of $2 \mathrm{~mm}$ from the tumor periphery, and then $25 \mu 1$ indigocarmine was subcutaneously injected. The other $25 \mu 1$ indigocarmine was subcutaneously injected into the rostral side by the same method. Consequently, the tissue around the tumor was stained blue (Fig. 1), and the injected solution did not indicate any leakage from the injection points.

Based on the aforementioned method, the mice were treated by peritumoral injection when the tumor mass reached $\sim 40 \mathrm{~mm}^{3}$ in size. Briefly, $25 \mu \mathrm{l}$ of $10 \mathrm{mg} / \mathrm{ml}$ bevacizumab or saline was subcutaneously injected into the caudal and rostral sides, respectively) using the Hamilton microliter syringe. Each $25 \mu \mathrm{l}$ from both sides (a total of $50 \mu \mathrm{l}$ ) was administered twice a week for 4 weeks. The mice in the control group did not receive any treatment.

Tumor diameters were measured twice a week for 28 days. Tumor volume was determined using the following formula: $\left(0.5 \mathrm{x}\right.$ length $\mathrm{x}$ width $\left.{ }^{2}\right)$, as described by Fujita et al (16). Mice were then euthanized and the tumor was carefully removed along with the overlying skin and the surrounding tissue (Fig. 2). Whole tumor specimens were fixed immediately with 4\% paraformaldehyde and embedded in paraffin for subsequent histological examinations. All animal experiments were approved by the Animal Ethics Committee of the University of Fukui (approval no. 28075) and performed in accordance with the Guide for the Care and Use of Laboratory Animals (National Institutes of Health) (23).

Immunohistochemistry. CD31 is mainly expressed in the vascular endothelia. In addition, $\alpha$-SMA is found in perivascular interstitial cells of microvascular proliferation. Thus, immunoreactivity to CD31 and $\alpha$-SMA shows the appearance of immature and mature tumor vessels, respectively (24). In order to evaluate the endothelial cells of intratumoral microvessels and the recruitment of vascular mural cells, immunostaining for CD31 and $\alpha$-SMA was performed. The 
A

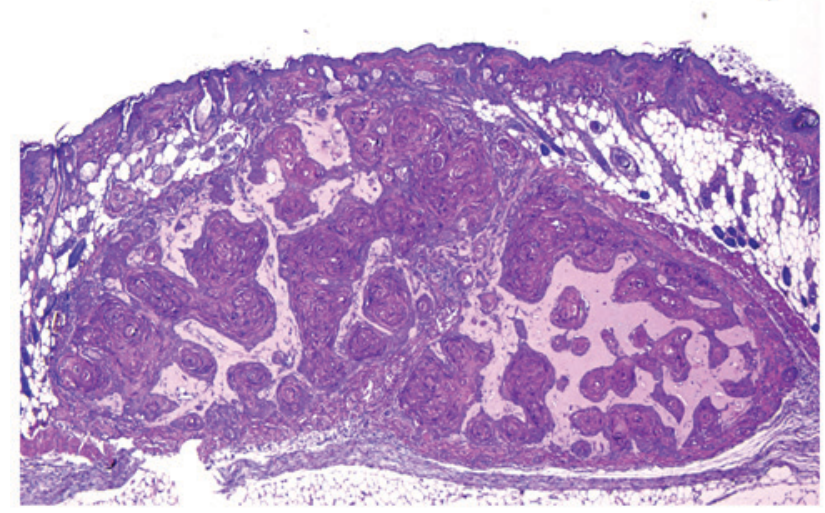

B

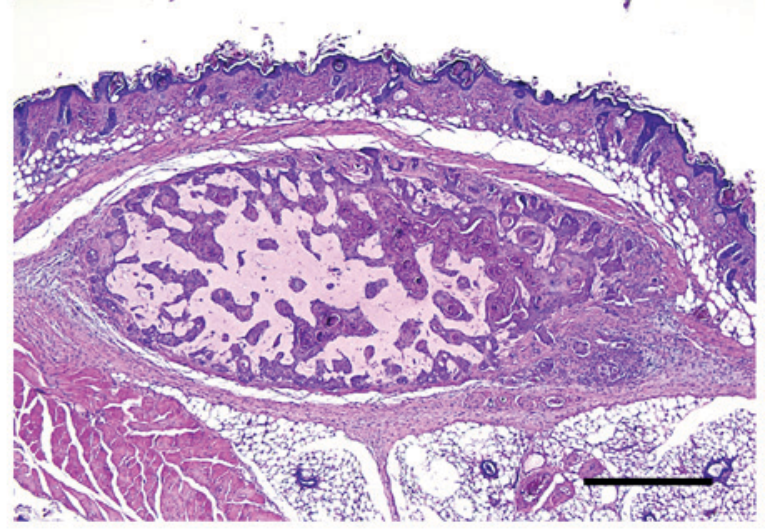

Figure 2. Representative image of the tumor (A) in the control group (28 days following tumor transplantation) and (B) in the bevacizumab-treated group. The tumor was removed along with the overlying skin and the surrounding tissue. Scale bar=500 $\mu \mathrm{m}$.

sections were de-paraffinized in Clear Plus (Falma Co., Ltd., Tokyo, Japan) for $15 \mathrm{~min}$ and rehydrated in $100 \%$ ethanol for $15 \mathrm{~min}$ at room temperature. The endogenous peroxide activity was eliminated with a solution of $0.3 \% \mathrm{H}_{2} \mathrm{O}_{2}$ in methanol for $15 \mathrm{~min}$ at room temperature. For antigen retrieval, the sections were immersed in $1 \mathrm{mM}$ EDTA-2Na/10 $\mathrm{mM}$ Tris buffer (pH 9.0) and autoclaved at $105^{\circ} \mathrm{C}$ for $15 \mathrm{~min}$. Protein Block Serum-Free solution (Dako; Agilent Technologies, Inc., Santa Clara, CA, USA; non-diluted) was used to block non-specific immunoreactions for $10 \mathrm{~min}$ at room temperature. The sections were incubated with primary antibodies against CD31 (cat no. DIA-310; DIANOVA Vertriebs Gesellschaft mbH; dilution, 1:20) and $\alpha$-SMA (cat no. ab5649; Abcam; dilution, 1:100) for $60 \mathrm{~min}$ at room temperature. The sections were then incubated with biotinylated rabbit anti-rat IgG (cat no. BA-4000; Vector Laboratories, Burlingame, CA, USA; dilution, 1:100) for CD31 or Histofine simple stain MAX-PO (cat. no. 424151; Nichirei Biosciences, Inc., Tokyo, Japan; non-diluted) for $\alpha$-SMA for $30 \mathrm{~min}$ at room temperature. This was followed by treatment with streptavidin-horseradish peroxidase for $60 \mathrm{~min}$ at room temperature (Vector Laboratories; dilution, 1:500). Immunoreactivity was visualized by immersing the sections in 3,3'-diaminobenzidine (DAB; DAB substrate kit; Dako; Agilent Technologies, Inc.). Subsequently, sections were counterstained with hematoxylin for $5 \mathrm{~min}$ at room temperature, dehydrated in $100 \%$ ethanol and replaced by Clear Plus at room temperature. The sections were rinsed three times in TBS between all steps. We observed them using a light microscope at magnification, $\mathrm{x} 400$.

Quantification of microvessel density (MVD) with CD31 and $\alpha$-SMA. CD31- and $\alpha$-SMA-stained tissue sections were analyzed, and all CD31- and $\alpha$-SMA-positive intratumoral microvessels were identified at magnification, $x 400$. These vessels were then counted in each individual microscopic field (x400), as described by Cao et al (17); at least 7 microscopic fields per histological section were included in the analysis. The results were presented as the average MVD per high power field.

Evaluation of apoptosis. The terminal deoxynucleotidyltransferase-mediated dUTP-biotin nick labeling (TUNEL) method was applied to evaluate apoptosis using the in situ Apoptosis Detection kit (Takara Bio, Inc., Otsu, Japan) according to the manufacturer's protocol. Briefly, the sections were de-paraffinized in Clear Plus for $15 \mathrm{~min}$, rehydrated in $100 \%$ ethanol for $15 \mathrm{~min}$ and permeabilized using $10 \mu \mathrm{g} / \mathrm{ml}$ proteinase K (Invitrogen; Thermo Fisher Scientific, Inc.) for $10 \mathrm{~min}$ at room temperature. The endogenous peroxide activity was eliminated with a solution of $3 \% \mathrm{H}_{2} \mathrm{O}_{2}$ for $5 \mathrm{~min}$ at room temperature. The sections were incubated with $50 \mu \mathrm{l}$ of labeling reaction mixture (consisting of TdT Enzyme $5 \mu 1+$ Labeling Safe Buffer $45 \mu \mathrm{l}$; Apoptosis Detection kit; Takara Bio, Inc., Otsu, Japan) in a $37^{\circ} \mathrm{C}$ humidified chamber for $90 \mathrm{~min}$. Then, they were reacted with $70 \mu \mathrm{l}$ anti-FITC horseradish peroxidase (not diluted, cat no. MK503; Takara Bio, Inc.) for $30 \mathrm{~min}$ at $37^{\circ} \mathrm{C}$. Immunoreactivity was visualized by immersing the sections in 3,3'-diaminobenzidine (DAB; Dako, Agilent Technologies, Inc., Santa Clara, CA, USA) for $10 \mathrm{~min}$ at room temperature. Subsequently, sections were counterstained with $3 \%$ methylgreen for $5 \mathrm{~min}$, dehydrated in $100 \%$ ethanol, and replaced by Clear Plus. EXCEL Mount (Falma, Tokyo, Japan) was used for mounting. The sections were rinsed three times in TBS between all steps. Apoptotic cells were counted at magnification, $\mathrm{x} 400$. The apoptosis index (the proportion of apoptotic cells) was determined by counting the TUNEL-positive cells per total number of tumor cells, avoiding necrotic tumor areas, from a minimum of 5 microscopic fields in each individual section using a light microscope.

Statistical analysis. All statistical analyses were performed using Excel Statistics 2012 software (version 1.13, Social Survey Research Information Co., Ltd., Tokyo, Japan; for windows). Measured values are presented as the mean \pm standard deviation (SD). Tumor volumes, MVD and apoptosis indices were analyzed using a one-way analysis of variance with the Tukey-Kramer multiple comparison post-hoc test. $\mathrm{P}<0.05$ was considered to indicate a statistically significant difference.

\section{Results}

Antitumor effects of bevacizumab on OSCC xenografts. The antitumor effects of bevacizumab on HSC-3 tumors 
Table I. Tumor volume in each group.

Tumor volume, $\mathrm{mm}^{3}$

\begin{tabular}{lccccr}
\cline { 2 - 5 } Treatment group & \multicolumn{1}{c}{0} & \multicolumn{1}{c}{7 days } & 14 days & 21 days & 28 days \\
\hline Control $(\mathrm{n}=6)$ & $58.9 \pm 6.4$ & $85.6 \pm 6.8$ & $88.7 \pm 18.7$ & $84.7 \pm 12.4$ & $102.2 \pm 17.6$ \\
Saline $(\mathrm{n}=6)$ & $62.2 \pm 7.9$ & $98.7 \pm 25.9$ & $79.0 \pm 15.5$ & $77.9 \pm 24.8$ & $94.6 \pm 10.5$ \\
Bevacizumab $(\mathrm{n}=6)$ & $70.2 \pm 29.5$ & $64.9 \pm 30.2$ & $40.0 \pm 10.6$ & $48.1 \pm 21.2$ & $57.1 \pm 28.4$
\end{tabular}

Tumor volume presented as mean $\pm \mathrm{SD}$.

propagated in nude mice were investigated. As indicated in Table I and Fig. 3, the tumor volume was reduced without any apparent toxicity in all bevacizumab-treated groups compared with the saline-treated groups and untreated controls. The differences between groups were statistically significant at 28 days following treatment $(\mathrm{P}<0.01)$.

Quantification of the density of CD31 and $\alpha$-SMA-positive microvessels in OSCC xenografts. In order to determine whether angiogenesis had been inhibited, the microvessel density in the xenograft tumors was examined by immunostaining for CD31. As demonstrated in Fig. 4, the densities of the CD31-positive microvessels in the untreated, saline-treated and bevacizumab-treated groups were $11.9 \pm 4.5,11.4 \pm 3.8$, and $6.4 \pm 3.0$, respectively. There was a significant difference in the densities of the CD31-positive microvessels between the bevacizumab-treated and the two control groups $(\mathrm{P}<0.01)$. To understand the recruitment of vascular mural cells, the densities of $\alpha$-SMA-positive microvessels were examined. As indicated in Fig. 5, the density of $\alpha$-SMA-positive microvessels in the untreated, saline-treated and bevacizumab-treated groups were $7.3 \pm 1.9,7.4 \pm 2.4$ and $3.7 \pm 1.5$, respectively. There was a significant difference in the densities of $\alpha$-SMA-positive microvessels between the bevacizumab-treated group and the two control groups $(\mathrm{P}<0.01)$.

Effects of bevacizumab treatment on apoptosis in OSCC xenografts. A histopathological analysis was performed with TUNEL staining, in order to examine apoptosis in the OSCC xenografts. As demonstrated in Fig. 6, the apoptosis indexes in the untreated, saline-treated and bevacizumab-treated groups were $0.6 \pm 1.0,0.7 \pm 0.9$ and $5.9 \pm 6.3 \%$, respectively. There was a significant difference in apoptosis levels between tumors treated with bevacizumab and tumors treated with either saline or left untreated $(\mathrm{P}<0.01)$.

\section{Discussion}

In the present study, the in vivo antitumor effects of bevacizumab were investigated in an OSCC xenograft model. It was identified that bevacizumab significantly inhibited tumor growth in the HSC-3-bearing nude mouse model. Histopathological analysis with CD31 staining of the xenograft samples indicated that bevacizumab treatment significantly decreased microvessel density compared to saline treatment or no treatment. These data are concordant with previous studies stating that treatment

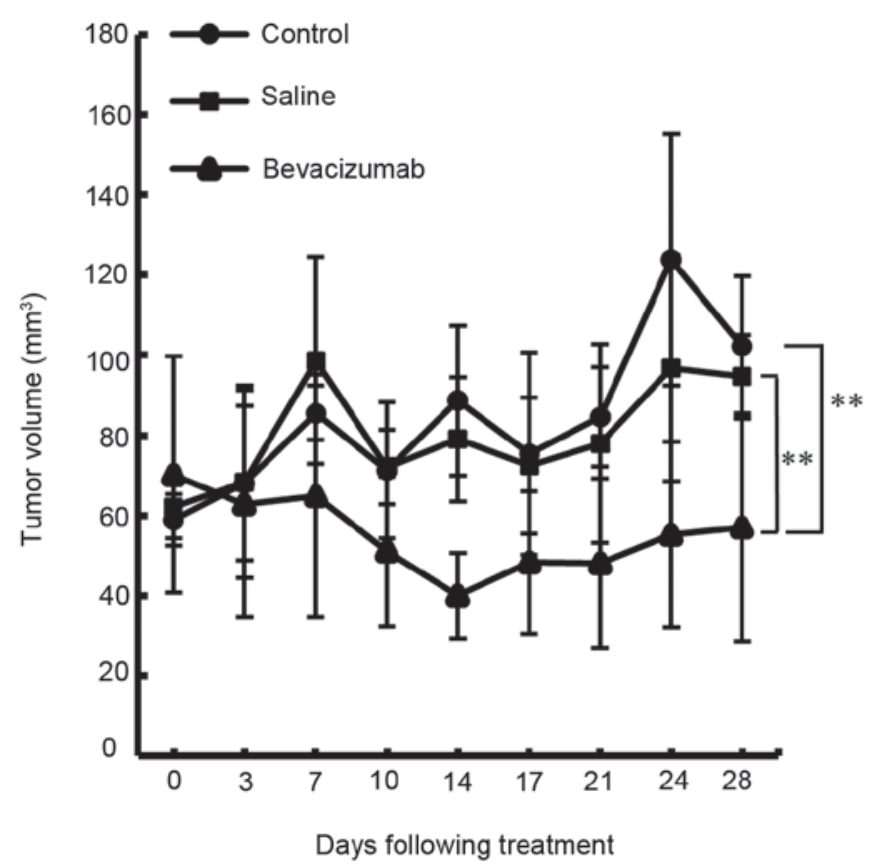

Figure 3. Anti-tumor effects of bevacizumab treatment in HSC-3 xenografts Following tumor formation, mice were grouped and treated with either saline $(n=6)$, bevacizumab $(n=6)$ or left untreated $(n=6)$. Tumor volumes were calculated as aforementioned. Each data point represents the mean tumor volume \pm SD. Inhibition of tumor growth in mice treated with bevacizumab was increased compared with that in mice treated with saline or left untreated $(\mathrm{P}<0.01) .{ }^{* * *} \mathrm{P}<0.01$.

with bevacizumab induced significant microvessel damage and tumor necrosis in HNSCC $(17,18)$. It was also identified that bevacizumab treatment significantly reduced the density of $\alpha$-SMA-positive microvessels compared with the control treatments. A previous study revealed that the expression ratio of $\alpha$-SMA/CD31 in a high VEGF group was significantly increased compared with that in a low VEGF group in glioblastoma multiforme (25). Furthermore, inhibition of VEGF by bevacizumab treatment significantly decreased the density of $\alpha$-SMA-positive microvessels in neuroblastoma compared with untreated control (26). These data support our hypothesis that bevacizumab treatment may mediate the suppression of immature and mature tumor vessels in an OSCC xenograft model.

The data of the present study also indicate that peritumoral bevacizumab injections have significant antitumor effects in an OSCC xenograft model. It is well known that local administration enhances antitumor effects in the treatment of SCC. 
A

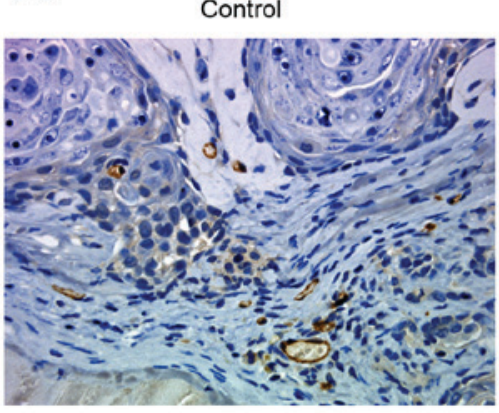

Saline

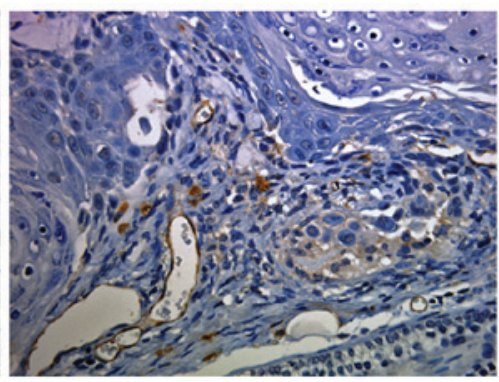

Bevacizumab

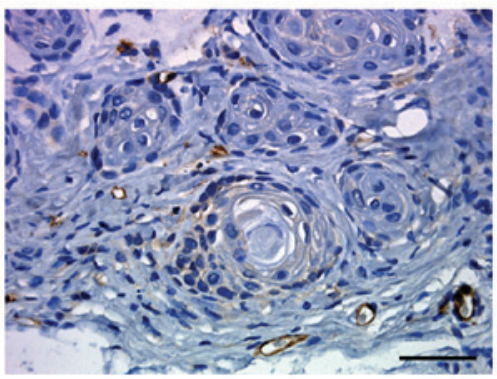

B

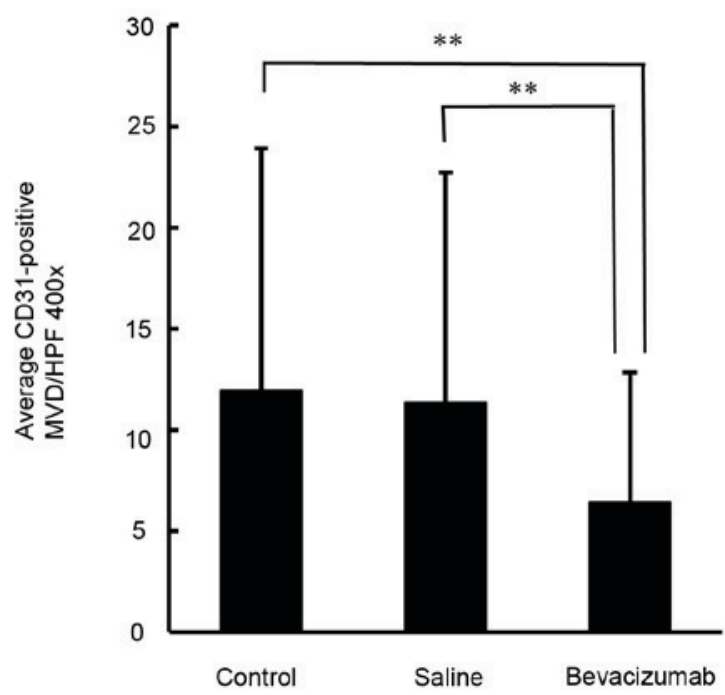

Figure 4. Immunohistochemical assessment of CD31-positive microvessel density in xenograft tumors in response to bevacizumab treatment. (A) Representative microphotographs of CD31-immunostained tumor sections obtained from untreated control mice and from mice treated with bevacizumab or saline. Scale bar=50 $\mu \mathrm{m}$. (B) Quantification of intratumoral microvessel density by CD31 immunostaining. Each bar represents the mean intratumoral microvessel density count \pm SD. A significant decrease in microvessel density was observed in the tumors treated with bevacizumab compared with untreated control or saline-treated tumors $(\mathrm{P}<0.01) .{ }^{* *} \mathrm{P}<0.01$.

For example, local injection of OK-432, a penicillin-killed and lyophilized preparation of a low-virulence strain of Streptococcus pyogenes (group A), was revealed to be effective in the treatment of SCC, including OSCC and esophageal carcinoma (27,28). Yamada et al (29) confirmed that local OK-432 injection induced tumor necrosis in SCC mouse models. In the present study, it was identified that apoptosis was significantly induced in xenograft tumors treated with bevacizumab as compared with those treated with either saline or left untreated. Other previous studies have also suggested that local administration of bevacizumab was able to induce apoptosis in SCC xenografts $(16,18,19)$, indicating that it may be beneficial for the induction of apoptosis in OSCC.

The primary purpose of the present study was to evaluate inhibition of angiogenesis by peritumoral injection of bevacizumab. To examine the microvessels around the tumor, the tumor along with the overlying skin and the surrounding tissues was removed. Tumor growth was determined by estimated tumor volumes, not by the tumor weights. In their study on the photodynamic therapy for R3230AC mammary carcinoma, Gibson et al (30) compared the calculated tumor volumes by tumor width and height to the surgically removed tumor volumes by their water displacement (30). They confirmed that the difference between calculated tumor volumes and water replacement data was $\sim 20 \%$, and based on these data, they monitored tumor growth by calculating tumor volumes (30). Other studies have also used calculated tumor volume as an indicator of tumor growth $(16,17,19)$.

The experimental methods in the present study have some limitations. The present study focused on tumor angiogenesis and examined the densities of CD31- and $\alpha$-SMA-positive microvessels around the tumor, and the tissues removed from the mice included skin, subdermal and tumor tissues. Thus, it was difficult to perform specific experiments, including western blotting, to evaluate the protein expression associated with the antitumor effect of peritumoral bevacizumab injection.

In the present study, peritumoral injections were selected over intratumoral injections for the administration of bevacizumab. Dvorak and Gresser (31) also employed peritumoral injections to avoid the possibility of needle-induced injury caused by intratumoral injections, and indicated that peritumoral injections of interferon damaged tumor microvessels and markedly suppressed tumor growth in their mouse model. In addition, Marumo et al (32) demonstrated that peritumoral injections were superior to subcutaneous injections in their ability to inhibit tumor growth in a nude mouse model of renal 
A

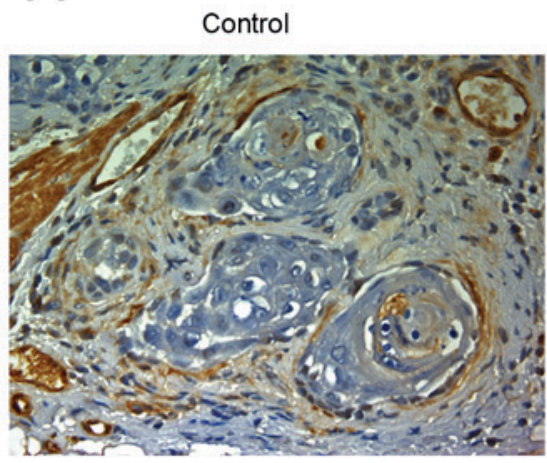

Saline

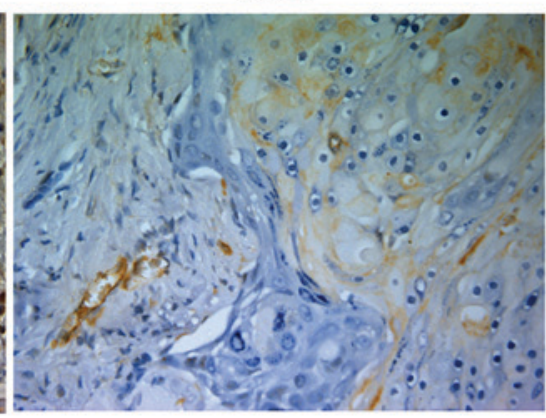

Bevacizumab

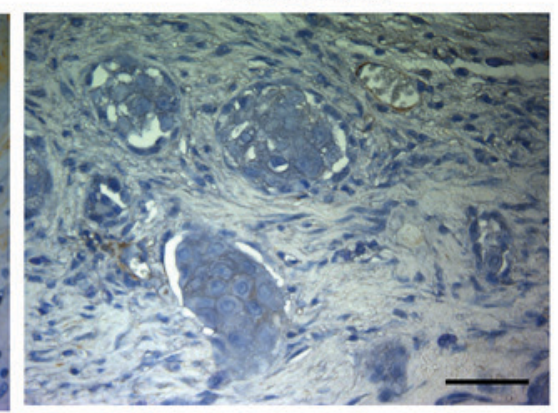

B

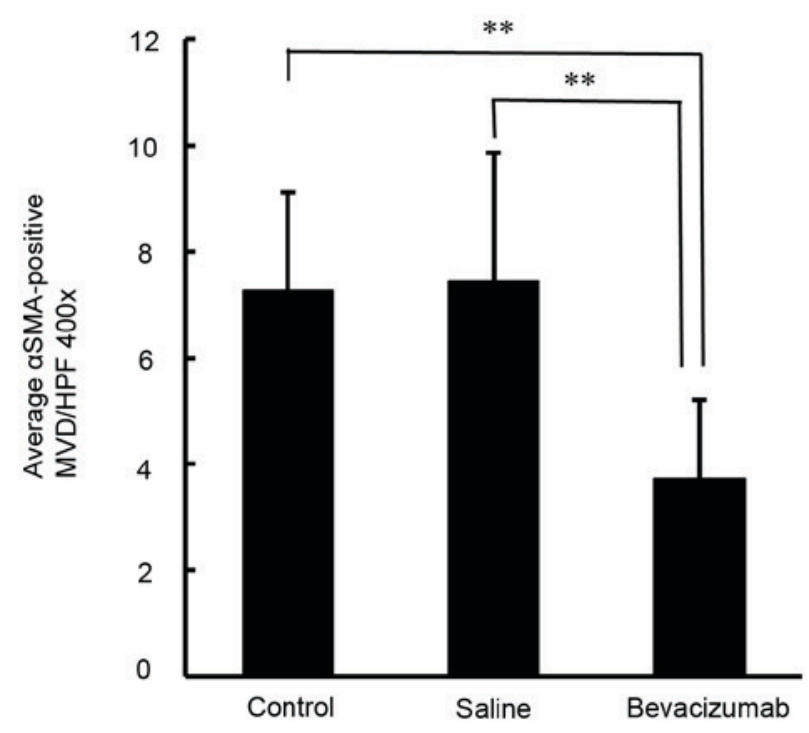

Figure 5. Immunohistochemical assessment of $\alpha$-SMA-positive microvessel density in xenograft tumors in response to bevacizumab treatment. (A) Representative microphotographs of $\alpha$-SMA-immunostained tumor sections obtained from untreated control mice and from mice treated with bevacizumab or saline. Scale bar $=50 \mu \mathrm{m}$. (B) Quantification of intratumoral microvessel density by $\alpha$-SMA immunostaining. Each bar represents the mean $\alpha$-SMA-positive intratumoral microvessel density count \pm SD. A significant decrease in $\alpha$-SMA-positive microvessel density was observed with bevacizumab treatment compared with the untreated control or saline-treated tumors $(\mathrm{P}<0.01)$. * $\mathrm{P}<0.01$. $\alpha$-SMA, $\alpha$-smooth muscle actin.

cell carcinoma. These data suggest that peritumoral injection is an effective method for the tumor treatments.

Regarding the method of peritumoral injection, a Hamilton microliter syringe with a 26-gauge needle was used in the present study. The needle was subcutaneously inserted a distance of $5 \mathrm{~mm}$ from the tumor periphery into the caudal side, positioned to a distance of $2 \mathrm{~mm}$ from the tumor periphery, and then $25 \mu \mathrm{l}$ bevacizumab or saline solution was injected. The other $25 \mu \mathrm{l}$ of the solution was injected into the rostral side by the same method. Consequently, the tumor was surrounded by the solution injected without any leakage. Yang et al (33) described a model in which macrophages were peritumorally injected at 4 locations surrounding the tumor mass. They tested intratumoral and peritumoral injections of macrophages, and found that the peritumoral injection was more efficient than intratumoral injection. Kitamura et al (34) described a method in which they administered ${ }^{125} \mathrm{I}$ monoclonal antibody peritumorally at one location (the distal portion) of the tumor in nude mice. It was concluded that antibody recruitment into the tumor was more efficient using the peritumoral injection than the intravenous injection. These 2 reports demonstrate that the peritumoral injection at 4 locations or one location surrounding the tumor was more effective than other injections, including the intratumoral and intravenous injection.

VEGF binds two primary tyrosine kinase receptors, VEGF receptor (VEGFR)-1 and VEGFR-2, in the vascular endothelium (35). The binding of VEGF with its receptors serves an important role in regulation of angiogenesis (35). VEGF expression was increased in tumor cells, and VEGF receptor expression was increased in CD31-positive endothelial cells in a mouse xenograft model of HNSCC (36). Henriques et al (37) demonstrated that VEGF was expressed in tumor and stromal cells in OSCC (37), and serum levels of VEGF were demonstrated to be increased in advanced T3/T4 tumors compared with early T1/T2 tumors (38), indicating that VEGF promotes tumor growth in OSCC. Conversely, bevacizumab is known to inhibit biological activity of VEGF (12). Therefore, peritumoral bevacizumab injection may have suppressed tumor angiogenesis and growth by inhibiting VEGF, which binds VEGF receptors in the tumor stroma (39), in this OSCC nude mouse xenograft model. However, 
A
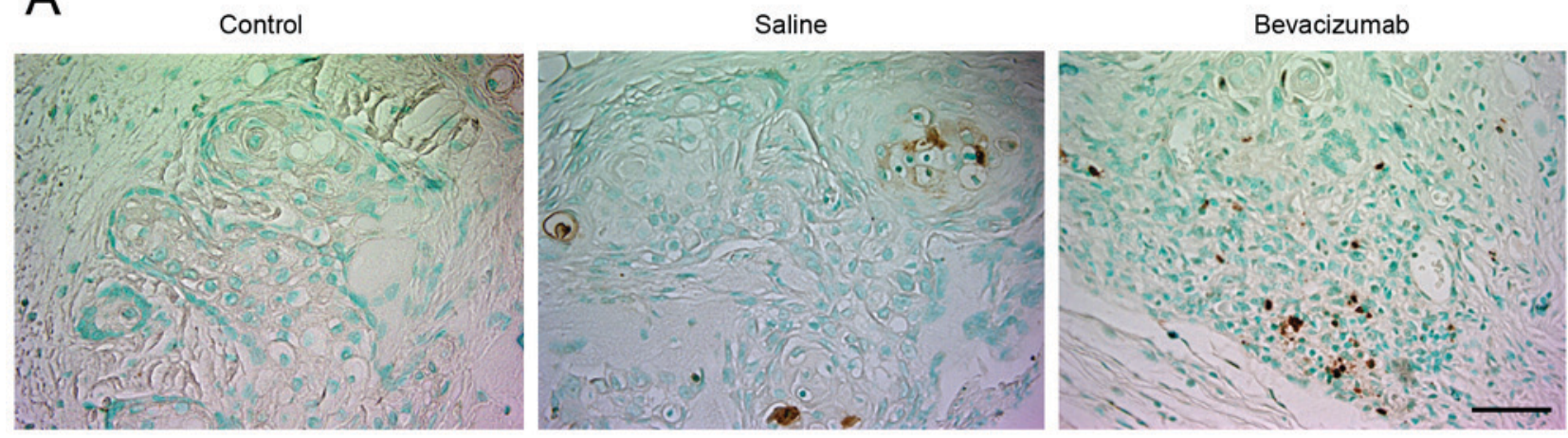

\section{B}

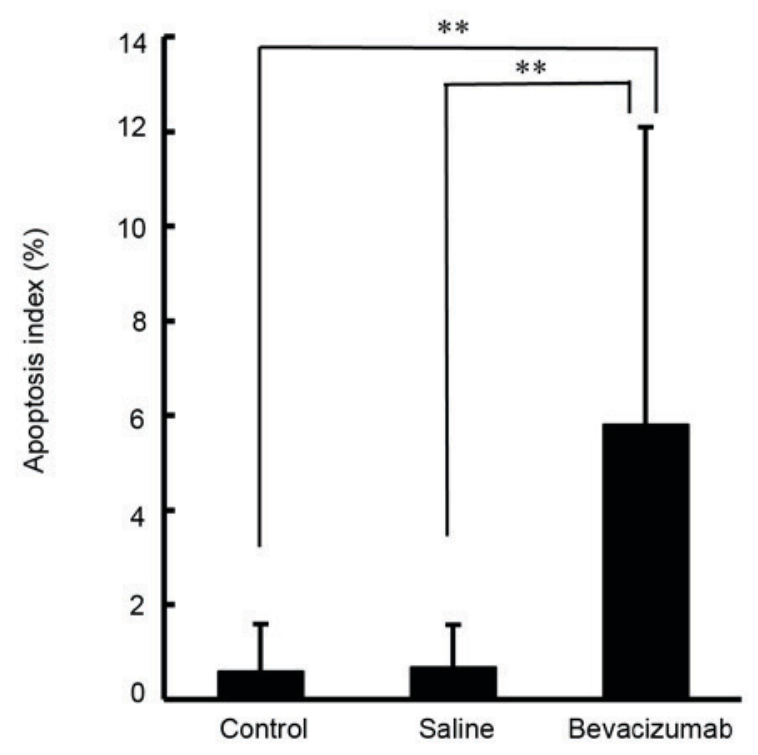

Figure 6. Analysis of apoptosis indexes in tumor xenografts in response to bevacizumab treatment. (A) Representative microphotographs of TUNEL staining (brown). Sections were obtained from untreated control mice and from mice treated with bevacizumab or saline. Scale bar=50 $\mu$ m. (B) Apoptosis index by TUNEL staining. Each bar represents the mean TUNEL-positive cell counts per total number of tumor cells \pm SD. A significant increase in the percentage of apoptotic cells was observed with bevacizumab treatment compared with untreated control or saline-treated tumors $(\mathrm{P}<0.01){ }^{* *} \mathrm{P}<0.01$. TUNEL, terminal deoxynucleotidyl-transferase-mediated dUTP-biotin nick labeling.

additional studies are required to confirm the mechanism of inhibiting tumor growth by peritumoral bevacizumab injection.

In conclusion, peritumoral bevacizumab injections were identified to inhibit tumor growth in OSCC xenografts. It was also demonstrated that treatment with bevacizumab significantly reduced the densities of the CD31- and $\alpha$-SMA-positive microvessels, and increased the tumor apoptosis index compared with the saline-treated or untreated groups. These data collectively suggest an experimental basis for the clinical development of peritumoral bevacizumab injections for the treatment of OSCC.

\section{Acknowledgements}

Not applicable.

\section{Funding}

The present study was supported by a Grant-in-Aid for Scientific Research (grant no. 26462999) from the Japan Society for the Promotion of Science, Japan.

\section{Availability of data and materials}

The datasets used and analyzed during the current study are available from the corresponding author on reasonable request.

\section{Authors' contributions}

HisY, HitY and KS designed the experiments. HisY, HitY, SM, TR, MK and KS performed the experiments. HisY, HitY, SM, TK, MK and KS analyzed the data. HisY, HitY and KS wrote the manuscript. All authors read and approved the final manuscript.

\section{Ethics approval and consent to participate}

All animal experiments were approved by the Animal Ethics Committee of the University of Fukui (approval no. 28075).

\section{Consent for publication}

Not applicable. 


\section{Competing interests}

The authors declare that they have no competing interests.

\section{References}

1. Torre LA, Bray F, Siegel RL, Ferlay J, Lortet-Tieulent J and Jemal A: Global cancer statistics, 2012. CA Cancer J Clin 65: 87-108, 2015

2. Braakhuis BJ, Leemans CR and Visser O: Incidence and survival trends of head and neck squamous cell carcinoma in the Netherlands between 1989 and 2011. Oral Oncol 50: 670-675, 2014.

3. Folkman J: Tumor angiogenesis: Therapeutic implications. N Engl J Med 285: 1182-1186, 1971.

4. Hsu HW, Wall NR, Hsueh CT, Kim S, Ferris RL, Chen CS and Mirshahidi S: Combination antiangiogenic therapy and radiation in head and neck cancers. Oral Oncol 50: 19-26, 2014

5. Vassilakopoulou M, Psyrri A and Argiris A: Targeting angiogenesis in head and neck cancer. Oral Oncol 51: 409-415, 2015.

6. Ferrara N, Gerber HP and LeCouter J: The biology of VEGF and its receptors. Nat Med 9: 669-676, 2003.

7. Christopoulos A, Ahn SM, Klein JD and Kim S: Biology of vascular endothelial growth factor and its receptors in head and neck cancer: Beyond angiogenesis. Head Neck 33: 1220-1229, 2011

8. Nayak S, Goel MM, Chandra S, Bhatia V, Mehrotra D, Kumar S, Makker A, Rath SK and Agarwal SP: VEGF-A immunohistochemical and mRNA expression in tissues and its serum levels in potentially malignant oral lesions and oral squamous cell carcinomas. Oral Oncol 48: 233-239, 2012.

9. Uehara M, Sano K, Ikeda H, Sekine J, Irie A, Yokota T, Tobita T, Ohba S and Inokuchi T: Expression of vascular endothelial growth factor and prognosis of oral squamous cell carcinoma. Oral Oncol 40: 321-325, 2004

10. Seki S, Fujiwara M, Matsuura M, Fujita S, Ikeda H, Asahina I and Ikeda T: Prediction of outcome of patients with oral squamous cell carcinoma using vascular invasion and the strongly positive expression of vascular endothelial growth factors. Oral Oncol 47: 588-593, 2011

11. Zhao SF, Yang XD, Lu MX, Sun GW, Wang YX, Zhang YK, $\mathrm{Pu}$ YM and Tang EY: Prognostic significance of VEGF immunohistochemical expression in oral cancer: A meta-analysis of the literature. Tumour Biol 34: 3165-3171, 2013

12. Presta LG, Chen H, O'Connor SJ, Chisholm V, Meng YG, Krummen L, Winkler M and Ferrara N: Humanization of an anti-vascular endothelial growth factor monoclonal antibody for the therapy of solid tumors and other disorders. Cancer Res 57: 4593-4599, 1997.

13. Hurwitz H, Fehrenbacher L, Novotny W, Cartwright T, Hainsworth J, Heim W, Berlin J, Baron A, Griffing S, Holmgren E, et al: Bevacizumab plus irinotecan, fluorouracil, and leucovorin for metastatic colorectal cancer. N Engl J Med 350: 2335-2342, 2004

14. Dickler MN, Rugo HS, Eberle CA, Brogi E, Caravelli JF, Panageas KS, Boyd J, Yeh B, Lake DE, Dang CT, et al: A phase II trial of erlotinib in combination with bevacizumab in patients with metastatic breast cancer. Clin Cancer Res 14: 7878-7883, 2008.

15. Sandler A, Gray R, Perry MC, Brahmer J, Schiller JH, Dowlati A, Lilenbaum R and Johnson DH: Paclitaxel-carboplatin alone or with bevacizumab for non-small-cell lung cancer. N Engl J Med 355: 2542-2550, 2006.

16. Fujita K, Sano D, Kimura M, Yamashita Y, Kawakami M, Ishiguro Y, Nishimura G, Matsuda $\mathrm{H}$ and Tsukuda M: Anti-tumor effects of bevacizumab in combination with paclitaxel on head and neck squamous cell carcinoma. Oncol Rep 18: 47-51, 2007.

17. Cao S, Durrani FA, Toth K, Rustum YM and Seshadri M: Bevacizumab enhances the therapeutic efficacy of Irinotecan against human head and neck squamous cell carcinoma xenografts. Oral Oncol 47: 459-466, 2011.

18. Gyanchandani R, Sano D, Ortega Alves MV, Klein JD, Knapick BA, Oh S, Myers JN and Kim S: Interleukin-8 as a modulator of response to bevacizumab in preclinical models of head and neck squamous cell carcinoma. Oral Oncol 49: 761-770, 2013.

19. Wang Y, Dong L, Bi Q, Ge X, Zhang X, Wu D, Fu J, Zhang C, Wang $\mathrm{C}$ and $\mathrm{Li} \mathrm{S}$ : Beyond antiangiogenesis: Intratumorally injected bevacizumab plays a cisplatin-sensitizing role in squamous cell carcinomas in mice. Chemotherapy 57 : 244-252, 2011.
20. Yancik R and Ries LA: Cancer in older persons: An international issue in an aging world. Semin Oncol 31: 128-136, 2004.

21. Argiris A, Li Y, Murphy BA, Langer CJ and Forastiere AA Outcome of elderly patients with recurrent or metastatic head and neck cancer treated with cisplatin-based chemotherapy. J Clin Oncol 22: 262-268, 2004.

22. Takahashi T, Ueda S, Kono K and Majima S: Attempt at local administration of anticancer agents in the form of fat emulsion. Cancer 38: 1507-1514, 1976.

23. National Research Council: Guide for the Care and Use of Laboratory Animals. 8th edition. The National Academies Press, Washington, DC, 2011. https://grants.nih.gov/grants/olaw/guidefor-the-care-and-use-of-laboratory-animals.pdf.

24. Sorace AG, Quarles CC, Whisenant JG, Hanker AB, McIntyre JO, Sanchez VM and Yankeelov TE: Trastuzumab improves tumor perfusion and vascular delivery of cytotoxic therapy in a murine model of HER $2^{+}$breast cancer: Preliminary results. Breast Cancer Res Treat 155: 273-284, 2016.

25. Takeuchi H, Hashimoto N, Kitai R, Kubota T and Kikuta K: Proliferation of vascular smooth muscle cells in glioblastoma multiforme. J Neurosurg 113: 218-224, 2010.

26. Patterson DM, Gao D, Trahan DN, Johnson BA, Ludwig A, Barbieri E, Chen Z, Diaz-Miron J, Vassilev L, Shohet JM and Kim ES: Effect of MDM2 and vascular endothelial growth factor inhibition on tumor angiogenesis and metastasis in neuroblastoma. Angiogenesis 14: 255-266, 2011

27. Mukai M, Kubota S, Morita S and Akanuma A: A pilot study of combination therapy of radiation and local administration of OK-432 for esophageal cancer. Five-year survival and local control rate. Cancer 75: 2276-2280, 1995.

28. Tano T, Okamoto M, Kan S, Bando T, Goda H, Nakashiro K, Shimodaira S, Koido S, Homma S, Fujita T, et al: Immunochemoradiotherapy for patients with oral squamous cell carcinoma: Augmentation of OK-432-induced helper T cell 1 response by 5-FU and X-ray irradiation. Neoplasia 15: 805-814, 2013.

29. Yamada T, Hayashi Y, Kaneko R, Tohnai I, Ueda M and Ito M Effect of the combination of a local OK-432 injection and hyperthermia on SCC VII tumors in mice. J Radiat Res 39: 101-109, 1998.

30. Gibson SL, VanDerMeid KR, Murant RS, Raubertas RF and Hilf R: Effects of various photoradiation regimens on the antitumor efficacy of photodynamic therapy for R3230AC mammary carcinomas. Cancer Res 50: 7236-7241, 1990.

31. Dvorak HF and Gresser I: Microvascular injury in pathogenesis of interferon-induced necrosis of subcutaneous tumors in mice. J Natl Cancer Inst 81: 497-502, 1989.

32. Marumo K, Oya M and Murai M: Application of the interferon minipellet to human renal cell carcinoma in nude mice. Int $\mathrm{J}$ Urol 4: 55-61, 1997.

33. Yang TD, Choi W, Yoon TH, Lee KJ, Lee JS, Joo JH, Lee MG, Yim HS, Choi KM, Kim B, et al: In vivo photothermal treatment by the peritumoral injection of macrophages loaded with goldnanoshells. Biomed Opt Express 7: 185-193, 2015

34. Kitamura K, Takahashi T, Kotani T, Miyagaki T, Yamaoka N, Tsurumi H, Noguchi A and Yamaguchi T: Local administration of monoclonal antibody-drug conjugate: A new strategy to reduce the local recurrence of colorectal cancer. Cancer Res 52: $6323-6328,1992$

35. Kliche $\mathrm{S}$ and Waltenberger J: VEGF receptor signaling and endothelial function. IUBMB Life 52: 61-66, 2001.

36. Yazama H, Kitatani K, Fujiwara K, Kato M, Hashimoto-Nishimura M, Kawamoto K, Hasegawa K, Kitano H, Bielawska A, Bielawski J and Okazaki T: Dietary glucosylceramides suppress tumor growth in a mouse xenograft model of head and neck squamous cell carcinoma by the inhibition of angiogenesis through an increase in ceramide. Int J Clin Oncol 20: 438-446, 2015.

37. Henriques AC, de Matos FR, Galvão HC and Freitas Rde A: Immunohistochemical expression of MMP-9 and VEGF in squamous cell carcinoma of the tongue. J Oral Sci 54: 105-111, 2012.

38. Aggarwal S, Devaraja K, Sharma SC and Das SN: Expression of vascular endothelial growth factor (VEGF) in patients with oral squamous cell carcinoma and its clinical significance. Clin Chim Acta 436: 35-40, 2014.

39. Folkman J: Role of angiogenesis in tumor growth and metastasis. Semin Oncol 29 (Suppl 16): S15-S18, 2002. 